\title{
Rashba and Dresselhaus spin splittings in semiconductor quantum wells measured by spin photocurrents
}

\author{
S. Giglberger, ${ }^{1}$ L. E. Golub, ${ }^{2}$ V. V. Bel'kov, ${ }^{2}$ S. N. Danilov, ${ }^{1}$ D. Schuh, ${ }^{1}$ C. Gerl, ${ }^{1}$ F. Rohlfing, ${ }^{1}$ J. Stahl, ${ }^{1}$ W. Wegscheider, ${ }^{1}$ \\ D. Weiss, ${ }^{1}$ W. Prettl, ${ }^{1}$ and S. D. Ganichev ${ }^{1}$ \\ ${ }^{1}$ Fakultät Physik, University of Regensburg, 93040, Regensburg, Germany \\ ${ }^{2}$ A.F. Ioffe Physico-Technical Institute, Russian Academy of Sciences, 194021 St. Petersburg, Russia
}

(Received 21 September 2006; published 18 January 2007)

\begin{abstract}
The spin-galvanic effect and the circular photogalvanic effect induced by terahertz radiation are applied to determine the relative strengths of Rashba and Dresselhaus band spin splitting in (001)-grown GaAs and InAs based two dimensional electron systems. We observed that shifting the $\delta$-doping plane from one side of the quantum well to the other results in a change of sign of the photocurrent caused by Rashba spin splitting while the sign of the Dresselhaus term induced photocurrent remains. The measurements give the necessary feedback for technologists looking for structures with equal Rashba and Dresselhaus spin splittings or perfectly symmetric structures with zero Rashba constant.
\end{abstract}

DOI: 10.1103/PhysRevB.75.035327

PACS number(s): 73.21.Fg, 72.25.Fe, 78.67.De, 73.63.Hs

\section{INTRODUCTION}

In low dimensional structures based on III-V compound semiconductors the spin degeneracy of the energy bands is removed. This lifting of spin degeneracy is caused by spinorbit interaction and results in terms linear in electron wave vector $\boldsymbol{k}$ in the effective Hamiltonian. The spin splitting is crucial for the field of spintronics, indeed it allows the electric field control of spin polarization, determines the spin relaxation rate, and can be utilized for all-electric spin injection. ${ }^{1}$ The microscopic origin of terms linear in electron wave vector in low dimensional systems is structure inversion asymmetry (SIA) and bulk inversion asymmetry (BIA) which lead to Rashba and Dresselhaus spin-orbit terms in the Hamiltonian, respectively. ${ }^{2-4}$ These terms can interfere resulting in an anisotropy of spin splitting and can even cancel each other if Rashba and Dresselhaus terms have equal strength resulting in a vanishing spin splitting in certain $\boldsymbol{k}$-space directions. ${ }^{5}$ This cancellation leads to new macroscopic effects such as disappearance of antilocalization, ${ }^{6}$ the absence of spin relaxation in specific crystallographic directions, ${ }^{7,8}$ the lack of Shubnikov-de Haas beating, ${ }^{9}$ and can be employed for a nonballistic spin-field effect transistor. ${ }^{10}$ Thus, the knowledge of the relative strength of Rashba and Dresselhaus terms is important for investigations of spin dependent phenomena in low dimensional structures. Recently we demonstrated ${ }^{11}$ that the spin-galvanic effect $(\mathrm{SGE})^{12-14}$ can be used as a tool to measure the ratio of Rashba and Dresselhaus terms (RD ratio) in a very direct way which does not rely on theoretically obtained quantities. Investigation of the angular dependence of the spin-galvanic current in InAs quantum wells, excited by terahertz radiation, allows us to deduce the RD ratio directly from experiment. In the present paper we apply this method to various (001)-oriented $n$-type InAs- and GaAs-based heterostructures and extended this novel technique by employing the circular photogalvanic effect (CPGE) ${ }^{5,13-18}$

\section{SPIN PHOTOCURRENTS AS A METHOD}

A direct way to explore the BIA and SIA terms in the Hamiltonian, which requires no knowledge of microscopic details, is based on the phenomenological equivalence of different mechanisms linearly coupling a polar vector such as wave vector or current with an axial-vector-like spin of electrons or angular momentum of photons. Indeed, such phenomena are described by second rank pseudotensors whose irreducible components differ by a scalar factor only. Thus, the anisotropy in space is the same for all such phenomena. The strength of the spin splitting in various crystallographic directions is described by Rashba and Dresselhaus terms in the Hamiltonian $H_{\mathrm{SO}}=\Sigma \beta_{l m} \sigma_{l} k_{m}$, where $\beta_{l m}$ is a second rank pseudotensor and $\sigma_{l}$ is the Pauli spin matrix. Nonzero components of second rank pseudotensors may exist in gyrotropic point groups only, where, by definition, axial and polar vector components transform equivalently by all symmetry operations. Thus the linear coupling between these vectors becomes possible. There are two experimentally accessible effects which are also described by such second rank pseudotensors: the spin-galvanic effect ${ }^{12} j_{\alpha}=\Sigma Q_{\alpha \beta} S_{\beta}$ and the circular photogalvanic effect ${ }^{15} j_{\alpha}=\Sigma \gamma_{\alpha \beta} \hat{e}_{\beta} P_{\text {circ }}$. Here $S$ is the average spin, $P_{\text {circ }}$ is a helicity of radiation, and $\hat{\boldsymbol{e}}$ is the projection of the unit vector pointing in the direction of light propagation onto the plane of the sample. In analogy to the band spin splitting and based on the equivalence of the invariant irreducible components of the pseudotensors $\beta, Q$, and $\gamma$, these currents can be decomposed into Rashba and Dresselhaus contributions which can be measured separately. Taking the ratio between these contributions cancels the scalar factor which contains all microscopic details. ${ }^{11}$ Therefore the ratio is constant for all mechanisms and photocurrents and can be used to determine the anisotropy of the spin splitting which is otherwise experimentally not easily accessible.

\section{A. Band structure}

First we briefly summarize the consequences of Rashba and Dresselhaus terms on the electron dispersion and on the spin orientation of the 2DEG's electronic eigenstates. We consider QWs of zinc-blende structure grown in [001] direction, belonging to $C_{2 v}$ point-group symmetry. In this point group the pseudotensor $\beta_{l m}$ has two nonvanishing invariant 

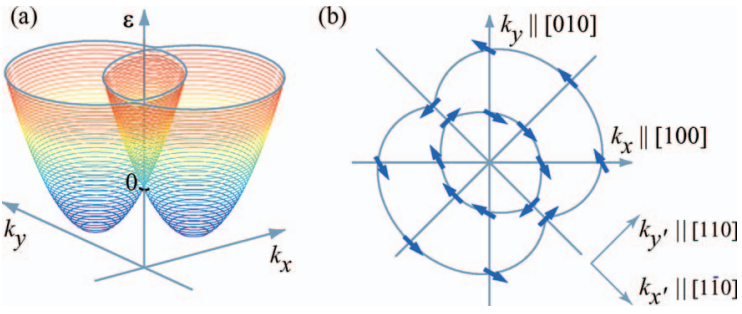

FIG. 1. (Color) Schematic 2D band structure with $\boldsymbol{k}$-linear terms for $C_{2 v}$ symmetry for nonequal strength of SIA and BIA. (a) The energy $\varepsilon$ as a function of $k_{x}$ and $k_{y}$, (b) the distribution of spin orientations at the 2D Fermi energy.

irreducible components $\alpha=\beta_{x y}=-\beta_{y x}$ and $\beta=\beta_{x x}=-\beta_{y y}$ which yield Rashba and Dresselhaus terms, respectively. The coordinate system used here is along the cubic axes of the crystal $x\|[100], y\|[010]$. The corresponding spin-orbit part $H_{\text {SO }}$ of the ground electron subband Hamiltonian

$$
H=\hbar^{2} k^{2} / 2 m^{*}+H_{\mathrm{SO}}
$$

( $m^{*}$ is the effective electron mass) contains a Rashba term as well as a Dresselhaus term according to

$$
H_{\mathrm{SO}}=\alpha\left(\sigma_{x} k_{y}-\sigma_{y} k_{x}\right)+\beta\left(\sigma_{x} k_{x}-\sigma_{y} k_{y}\right) .
$$

The energy spectrum of such systems consists of two branches with the following anisotropic dispersions: ${ }^{19}$

$$
\varepsilon_{ \pm}(\boldsymbol{k})=\frac{\hbar^{2} k^{2}}{2 m^{*}} \pm k \sqrt{\alpha^{2}+\beta^{2}+2 \alpha \beta \sin 2 \vartheta_{k}},
$$

where $\vartheta_{\boldsymbol{k}}$ is the angle between $\boldsymbol{k}$ and the $x$ axis.

To illustrate the resulting energy dispersion we plot in Fig. 1 the eigenvalues of $H, \varepsilon(\boldsymbol{k})$ (left panel), and contours of constant energy in the $k_{x}, k_{y}$ plane together with the spin orientation of eigenstates at selected points in $\boldsymbol{k}$ space (right panel). Here we assumed that $\alpha>\beta>0$, and $\alpha / \beta=1.15$. Rashba and Dresselhaus terms result in different patterns of spin eigenstates in $\boldsymbol{k}$ space. The distribution of spin directions in $\boldsymbol{k}$ space can be visualized by writing the spin-orbit interaction term in the form $H_{\mathrm{SO}}=\hbar \sigma \cdot \Omega_{\mathrm{eff}}(\boldsymbol{k})$, where $\Omega_{\mathrm{eff}}(\boldsymbol{k})$ is an effective Larmor precession frequency providing the relevant quantization axis. In the presence of both Rashba and Dresselhaus spin-orbit couplings, characteristic for $C_{2 v}$ symmetry, the $[1 \overline{1} 0]$ and the $[110]$ axes become nonequivalent (see Fig. 1). The maximum and minimal spin splittings take place along these axes and are equal to $2(\alpha+\beta) k$ and $2(\alpha-\beta) k$, respectively.

The role of $\boldsymbol{k}$-cubic spin-orbit splitting. So far we discussed $k$-linear terms in the Hamiltonian only. In fact, in zinc-blende structure based (001)-grown quantum wells also terms cubic in $k$ are present which stem from the Dresselhaus term in the host bulk material. The corresponding effective precession frequency $\Omega_{\mathrm{eff}} \propto k^{3}$ in quantum wells can be conveniently decomposed as $\Omega_{\text {eff }}=\Omega_{1}+\Omega_{3}$, where $\Omega_{1}$ varies with the angle $\vartheta_{k}$ as combinations of $\cos \vartheta_{k}$, $\sin \vartheta_{k}$, and $\Omega_{3}$ as $\cos 3 \vartheta_{k}, \sin 3 \vartheta_{k}$ terms (see, for instance, Refs. ${ }^{20,21}$ ).
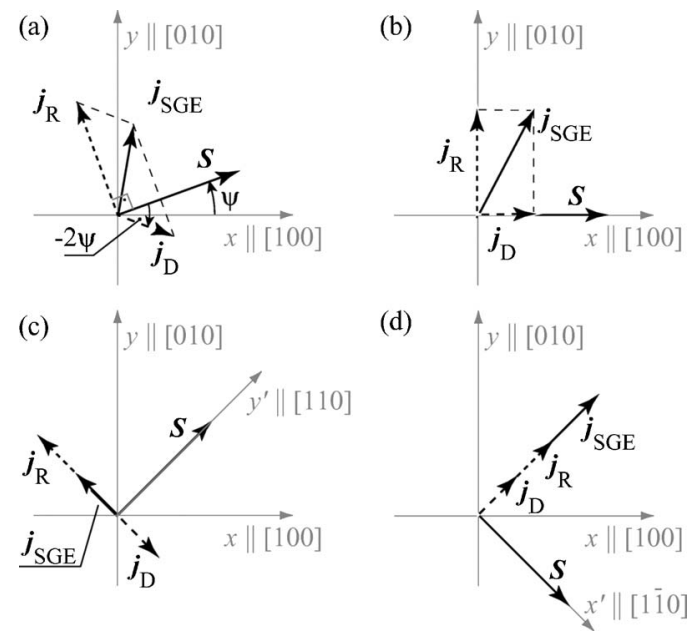

FIG. 2. Spin-galvanic currents in an (001)-grown QW for inplane average spin direction given by arbitrary angle $\Psi$ (a), SGE-I geometry (b), and SGE-II geometry (c) and (d). It is assumed that $\alpha>\beta>0$.

Both SGE and CPGE are caused by the term $\Omega_{1}$ only. Hence, the Dresselhaus term in the Hamiltonian, which yields the photocurrent, is given by the sum of the term linear in $\boldsymbol{k}$ discussed above and the terms cubic in $\boldsymbol{k}$ given by $\Omega_{1}$. The last terms only renormalize the Dresselhaus parameter $\beta$ which should be replaced by $\beta-\gamma k^{2} / 4$. Here $\gamma$ is the bulk spin-orbit constant. The cubic terms given by $\Omega_{3}$ do not result in spin photocurrents, however, they modify the spin splitting and may affect spin relaxation and the anisotropy of spin-flip Raman scattering. ${ }^{21-25}$

\section{B. Spin-galvanic effect}

The spin-galvanic effect consists of the generation of an electric current due to a non-equilibrium spin polarization and is caused by asymmetric spin relaxation. ${ }^{5,12-14}$ The spingalvanic effect generally needs no optical excitation but may also occur due to optical spin orientation yielding a spin photocurrent. The SGE current $j_{\mathrm{SGE}}$ and the average spin are related by a second rank pseudo-tensor with components proportional to the parameters of spin-orbit splitting as follows:

$$
j_{\mathrm{SGE}}=A\left(\begin{array}{cc}
\beta & -\alpha \\
\alpha & -\beta
\end{array}\right) S,
$$

where $S$ is the average spin in the plane of the heterostructure and $A$ is a constant determined by the kinetics of the SGE, namely by the characteristics of momentum and spin relaxation processes.

Equation (3) allows one to determine the RD ratio. This is sketched in Fig. 2(a) showing the average spin $S$ and the spin-galvanic current $\boldsymbol{j}_{\mathrm{SGE}}$ which is decomposed into $\boldsymbol{j}_{R}$ and $\boldsymbol{j}_{D}$ proportional to the Rashba constant $\alpha$ and the Dresselhaus constant $\beta$, respectively.

By symmetry arguments the current $\boldsymbol{j}_{R}$ is always perpendicular to the average spin $S$ while the current $j_{D}$ encloses an angle $-2 \Psi$ with $S$, where $\Psi$ is the angle between $S$ and the 
[100] axis. The absolute value of the total current $j_{\mathrm{SGE}}$ is given by the expression

$$
j_{\mathrm{SGE}}=\sqrt{j_{R}^{2}+j_{D}^{2}-2 j_{R} j_{D} \sin 2 \Psi},
$$

which has the same algebraic form as the spin-orbit term in the band structure, see Eq. (2). Hence, by mapping the magnitude of the photocurrent in the plane of the QW the RD ratio $\alpha / \beta$ can be directly extracted from experiment. Two experimental geometries, SGE-I and SGE-II, are particularly suited to obtain $\alpha / \beta$.

Geometry SGE-I. In the first case $S$ is oriented along the $x$ axis parallel to the [100] direction $(\Psi=0)$. Then it follows from Eq. (4) that $\boldsymbol{j}_{D}$ and $\boldsymbol{j}_{R}$ are directed along and perpendicular to $S$, respectively. This situation is sketched in Fig. 2(b). The ratio of the currents measured along the $x$ and the $y$ axes gives

$$
\frac{\alpha}{\beta}=\frac{j_{y}(S \| x)}{j_{x}(S \| x)} .
$$

In addition to the ratio of two spin-splitting contributions, this geometry unambiguously shows whether the Rashba or Dresselhaus contribution is dominating. Furthermore, Eq. (5) allows one the experimental determination of not only the ratio but also the relative sign of the Rashba and Dresselhaus constants.

Geometry SGE-II. Figures 2(c) and 2(d) illustrate the second geometry. Here two measurements need to be carried out, one with $S$ along the $y^{\prime}$ axis parallel to the [110] direction $(\Psi=\pi / 4)$, and the second with $S$ along the $x^{\prime}$ axis parallel to the $[1 \overline{1} 0]$ direction $(\Psi=-\pi / 4)$. In both cases the total current flows perpendicularly to the spin. For the spin oriented along $y^{\prime}$ the Rashba and Dresselhaus current contributions are oppositely aligned and the total current has the magnitude $j_{x^{\prime}}\left(\boldsymbol{S} \| y^{\prime}\right)=j_{R}-j_{D}$. For the spin along $x^{\prime}$, however, the current contributions are collinear and the total current is given by $j_{y^{\prime}}\left(\boldsymbol{S} \| x^{\prime}\right)=j_{R}+j_{D}$. The relative strength of these currents

$$
r=\left|\frac{j_{x^{\prime}}\left(\boldsymbol{S} \| y^{\prime}\right)}{j_{y^{\prime}}\left(\boldsymbol{S} \| x^{\prime}\right)}\right|=\left|\frac{\alpha-\beta}{\alpha+\beta}\right|
$$

allows us a quantitative determination of the RD ratio via

$$
\frac{\alpha}{\beta}=\frac{1+r}{1-r} .
$$

Thus the geometry SGE-II gives information about the relative strength of the spin-splitting parameters, however, in contrast to the geometry SGE-I, it does not distinguish between the Rashba and Dresselhaus terms. This fact makes such a procedure not as useful as the geometry SGE-I. Here we use the geometry SGE-II for demonstration of selfconsistency of the method only. However, as we show below, this geometry is suitable for controllable growing of structures with equal Rashba and Dresselhaus constants.

\section{Circular photogalvanic effect}

The circular photogalvanic effect is another phenomenon which links the current to the spins plitting in heterostruc-
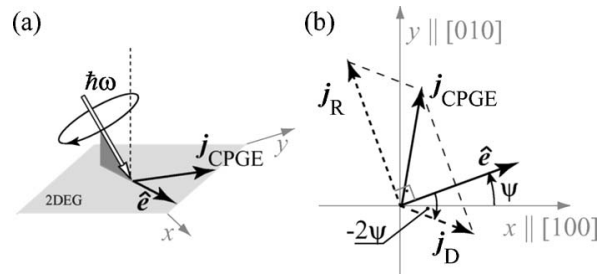

FIG. 3. Geometry of CPGE measurements (a) and angular dependence of the CPGE current (b).

tures. The CPGE is a result of selective photoexcitation of carriers in $\boldsymbol{k}$ space with circularly polarized light due to optical selection rules.,13-15 In (001)-grown two dimensional structures the CPGE current is generated by oblique illumination of the sample with circularly polarized light only 26,27 [see Fig. 3(a)]. The CPGE current $j_{\text {CPGE }}$ is proportional to the light's helicity $P_{\text {circ }}$ and depends on the direction of light propagation $\hat{\boldsymbol{e}}$ :

$$
\boldsymbol{j}_{\mathrm{CPGE}}=C\left(\begin{array}{cc}
\beta^{\prime} & -\alpha^{\prime} \\
\alpha^{\prime} & -\beta^{\prime}
\end{array}\right) \hat{\boldsymbol{e}} P_{\mathrm{circ}}
$$

where $C$ is the CPGE constant determined by the optical selection rules and by the momentum relaxation time, $\hat{\boldsymbol{e}} P_{\text {circ }} E_{0}^{2}=i\left(\boldsymbol{E} \times \boldsymbol{E}^{*}\right), \boldsymbol{E}$ is the complex amplitude of the electric field of the electromagnetic wave and $E_{0}=|\boldsymbol{E}|$ is the electric field amplitude. This equation shows that the CPGE current also consists of contributions proportional to Rashba and Dresselhaus constants $\alpha^{\prime}$ and $\beta^{\prime}$ as displayed in Fig. 3(b) by $\boldsymbol{j}_{R}$ and $\boldsymbol{j}_{D}$. In contrast to the SGE, CPGE current is determined by spin-splitting constants not only in the ground electronic subband but also by those in the excited conduction and valence size-quantized subbands. This is because the CPGE, caused by Drude absorption, is formed due to virtual transitions via other subbands and is hence determined by their spin-orbit splittings as well. This fact is indicated in Eq. (8) by using primed Rashba and Dresselhaus constants $\alpha^{\prime}$ and $\beta^{\prime}$. Due to symmetry arguments discussed above Eq. (8) has the same mathematical form as Eq. (3) which describes the spin-galvanic current. The physical content, however, is different which reflected by the different scalar factors $A$ and $C$ as well as by the different pseudovectors $S$ and $\hat{\boldsymbol{e}} P_{\text {circ. }}$. Due to this similarity the evaluation of the anisotropy of spin splitting obtained from CPGE follows exactly the same procedures as those for the spin-galvanic effect. The only step to be done is to replace the in-plane average spin $S$ by the pseudovector $\hat{\boldsymbol{e}} P_{\text {circ }}$.

Then in the geometry CPGE-I $\hat{\boldsymbol{e}}$ should be aligned along the $x$ axis parallel to the [100] direction $(\Psi=0)$ and the ratio of the currents measured along the $x$ and the $y$ axes gives

$$
\frac{\alpha^{\prime}}{\beta^{\prime}}=\left|\frac{j_{y}(\hat{\boldsymbol{e}} \| x)}{j_{x}(\hat{\boldsymbol{e}} \| x)}\right| .
$$

As for the geometry SGE-II for the geometry CPGE-II two measurements need to be carried out, one with the $\hat{\boldsymbol{e}}$ along the $y^{\prime}$ axis $(\Psi=\pi / 4)$, and the second with $\hat{\boldsymbol{e}}$ along the 


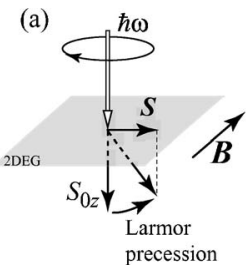

(b)

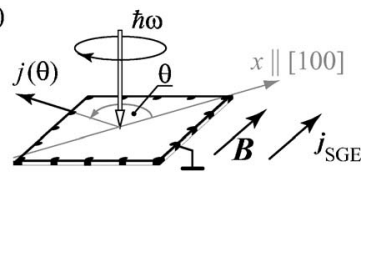

FIG. 4. Geometry of SGE measurements under optical excitation and Hanle effect (a) and angular dependence of the SGE current (b).

$x^{\prime}$-axis $(\Psi=-\pi / 4)$. In both cases the total current should be measured perpendicularly to $\hat{\boldsymbol{e}}$ and the RD ratio can be obtained from

$$
\frac{\alpha^{\prime}}{\beta^{\prime}}=\frac{1+r^{\prime}}{1-r^{\prime}},
$$

where

$$
r^{\prime}=\left|\frac{j_{x^{\prime}}\left(\hat{\boldsymbol{e}} \| y^{\prime}\right)}{j_{y^{\prime}}\left(\hat{\boldsymbol{e}} \| x^{\prime}\right)}\right| .
$$

Obviously, the CPGE-II method again does not distinguish between Rashba and Dresselhaus terms.

\section{Experimental}

As shown above the experimental access to the spin-orbit coupling parameters $\alpha$ and $\beta$ is provided by mapping the current in the plane of the heterostructure. Basically it is sufficient to measure either the spin-galvanic current or the circular photogalvanic effect in one of the geometries introduced above. However, to have a cross check and to increase the accuracy, redundant measurements for a number of crystallographic directions are desirable. The component of the total current along any in-plane direction given by the angle $\theta$ can be written as a sum of the projections of $\boldsymbol{j}_{R}$ and $\boldsymbol{j}_{D}$ onto this direction, following

$$
j(\theta)=j_{D} \cos (\theta+\Psi)+j_{R} \sin (\theta-\Psi) .
$$

Such measurements can be performed with a sample having large number of contacts around the edges of the specimen as shown in Fig. 4(b). The current is collected from opposite contacts one-by-one at different angles $\theta$.

\section{Spin-galvanic effect}

For the method based on the spin-galvanic effect one should know the direction of the average spin in the geometry SGE-I or the ratio between $S \| x^{\prime}$ and $S \| y^{\prime}$ in the geometry SGE-II [see Eq. (6)]. This is, however, not an easy task.

Using optical spin orientation and an external magnetic field $\boldsymbol{B}$ to generate the SGE, the necessary information about the average spin can be obtained from the direction of $\boldsymbol{B}$. The nonequilibrium in-plane average spin $S$ is prepared in the following way: Circularly polarized light at normal incidence on the QW plane polarizes the electrons in the lowest conduction subband resulting in monopolar spin orientation in the $z$ direction, $S_{0 z}$ in Fig. 4(a). An in-plane magnetic field rotates the spin to the QW plane. The competition of Larmor precession and spin relaxation results in the appearance of a steady state non-equilibrium in-plane average spin $\boldsymbol{S} \propto \boldsymbol{\omega}_{L} \tau_{S}$, where $\boldsymbol{\omega}_{L} \propto \boldsymbol{B}$ is the Larmor frequency, and $\tau_{s}$ the spin relaxation time. ${ }^{28}$ Finally the in-plane average spin $\boldsymbol{S}$ causes the spin-galvanic effect [Fig. 4(b)]. Applying a magnetic field $\boldsymbol{B}$ along $y$ we realize the situation similar the method SGE-I, and orienting $\boldsymbol{B}$ along $x^{\prime}$ and $y^{\prime}$ we obtain the geometry SGE-II. To obtain the RD ratio, however we should take into account a possible anisotropy of the spin relaxation.

Now let us consider, for instance, the geometry, where the magnetic field is oriented along $y$. The steady state spin components can be decomposed by projections on the axes $x^{\prime}$ and $y^{\prime}$ where the spin relaxation rate tensor is diagonal with the following components:

$$
S_{x^{\prime}}=-\frac{\omega_{L}}{\sqrt{2}} \tau_{s x^{\prime}} S_{0 z}, \quad S_{y^{\prime}}=\frac{\omega_{L}}{\sqrt{2}} \tau_{s y^{\prime}} S_{0 z},
$$

where $\tau_{s i}$ is the relaxation time for the $i$ th spin component.

Then it follows from Eqs. (3) and (13) that the ratio of the currents is given by 29,30

$$
\frac{j_{y}}{j_{x}}=\frac{\alpha\left(\tau_{s x^{\prime}}+\tau_{s y^{\prime}}\right)+\beta\left(\tau_{s x^{\prime}}-\tau_{s y^{\prime}}\right)}{\beta\left(\tau_{s x^{\prime}}+\tau_{s y^{\prime}}\right)+\alpha\left(\tau_{s x^{\prime}}-\tau_{s y^{\prime}}\right)} .
$$

In contrast to the geometry SGE-I, the ratio of the currents $j_{y} / j_{x}$ is not simply equal to $\alpha / \beta$ but is a function of the anisotropy of spin relaxation times.

There are two main spin relaxation mechanisms in III-V compound based heterostructures: the precessional one proposed by D'yakonov and Perel' and the scattering-induced mechanism of Elliott and Yafet. While Elliott-Yafet spin relaxation is isotropic in the heterostructure plane, the D'yakonov-Perel' mechanism is anisotropic as shown in Refs. 8 and 31. This difference affects the analysis of the SGE measurements and, on the other hand, allows one to discriminate the dominant spin relaxation mechanism in the studied system. In the case of a dominant Elliott-Yafet mechanism spin relaxation is isotropic, therefore $\tau_{s y^{\prime}}=\tau_{s x^{\prime}}$ and the spin points always normal to the magnetic field $(\boldsymbol{S} \perp \boldsymbol{B})$. Thus, the ratio of the Rashba and Dresselhaus spinsplittings in the geometry SGE-I is given by

$$
\frac{\alpha}{\beta}=\frac{j_{y}(\boldsymbol{B} \| y)}{j_{x}(\boldsymbol{B} \| y)} .
$$

If spin relaxation is governed by the D'yakonov-Perel' mechanism, however, spin relaxation is proved to be anisotropic ${ }^{7,8}$

$$
\frac{\tau_{s y^{\prime}}}{\tau_{s x^{\prime}}}=\left(\frac{\alpha-\beta}{\alpha+\beta}\right)^{2}
$$

This anisotropy needs to be taken into account yielding

$$
\frac{\alpha}{\beta}=\frac{\left(r^{\prime \prime}+1\right)^{1 / 3}+\left(r^{\prime \prime}-1\right)^{1 / 3}}{\left(r^{\prime \prime}+1\right)^{1 / 3}-\left(r^{\prime \prime}-1\right)^{1 / 3}},
$$

where 
TABLE I. Parameters of samples and measured RD ratios. Mobility and electron sheet density data are obtained at $4.2 \mathrm{~K}$ in the dark. We note that RD ratios are extracted from SGE measurements on a limited number of samples because in some structures the Dresselhaus SGE current has been masked by magnetogyrotropic currents (Ref. 32).

\begin{tabular}{lcccccccc}
\hline \hline Sample & material & $\begin{array}{c}\text { QW width } \\
\AA\end{array}$ & $\begin{array}{c}\text { spacer } 1 \\
\AA\end{array}$ & $\begin{array}{c}\text { spacer } 2 \\
\AA\end{array}$ & $\begin{array}{c}\text { mobility } \\
\left(\mathrm{cm}^{2} / \mathrm{Vs}\right)\end{array}$ & $\begin{array}{c}\text { density } \\
\mathrm{cm}^{-2}\end{array}$ & $\begin{array}{c}\alpha / \beta \\
\text { SGE }\end{array}$ & $\begin{array}{c}\alpha^{\prime} / \beta^{\prime} \\
\text { CPGE }\end{array}$ \\
\hline No. 1 & InAs/AlGaSb & 150 & & & $3.0 \times 10^{5}$ & $8 \times 10^{11}$ & 2.1 & 2.3 \\
No. 2 & InAs/AlGaSb & 150 & & & $2.0 \times 10^{5}$ & $1.4 \times 10^{12}$ & 1.8 \\
No. 3 & InAs/InAlAs & 60 & & 75 & $1.1 \times 10^{5}$ & $7.7 \times 10^{11}$ & 1.6 \\
No. 4 & GaAs/AlGaAs & $\infty$ & 700 & & $3.5 \times 10^{6}$ & $1.1 \times 10^{11}$ & 7.6 & 7.6 \\
No. 5 & GaAs/AlGaAs & 82 & 50 & 50 & $2.6 \times 10^{6}$ & $9.3 \times 10^{11}$ & -4.5 & -4.2 \\
No. 6 & GaAs/AlGaAs & 150 & 600 & 300 & $1.0 \times 10^{5}$ & $6.6 \times 10^{11}$ & -3.8 \\
No. 7 & GaAs/AlGaAs & 150 & 400 & 500 & $2.6 \times 10^{5}$ & $5.3 \times 10^{11}$ & -2.4 \\
No. 8 & GaAs/AlGaAs & 300 & 700 & & $3.2 \times 10^{6}$ & $1.3 \times 10^{11}$ & 2.8 \\
No. 9 & GaAs/AlGaAs & 300 & 700 & 1000 & $3.4 \times 10^{6}$ & $1.8 \times 10^{11}$ & & 1.5 \\
\hline \hline
\end{tabular}

$$
r^{\prime \prime}=\frac{j_{y}(\boldsymbol{B} \| y)}{j_{x}(\boldsymbol{B} \| y)} .
$$

Using the same argument we obtain $\alpha / \beta$ for $\boldsymbol{B}$ directed along $x^{\prime}$ or $y^{\prime}$ :

$$
\frac{j_{x^{\prime}}\left(\boldsymbol{B} \| x^{\prime}\right)}{j_{y^{\prime}}\left(\boldsymbol{B} \| y^{\prime}\right)}=\left(\frac{\alpha-\beta}{\alpha+\beta}\right)^{3} \text {. }
$$

\section{Circular photogalvanic effect}

The method based on CPGE measurements needs the knowledge of the light propagation direction which can be easily controlled. Then the RD ratio is directly obtained from the current measured in the geometry CPGE-I or CPGE-II making use of Eqs. (9) and (10), respectively.

\section{SAMPLES AND EXPERIMENTAL SETUP}

The experiments are carried out on (001)-oriented $n$-type GaAs/AlGaAs and InAs/AlGaSb heterostructures. Quantum well structures and single heterojunctions with various widths, carrier densities and mobilities are grown by molecular-beam epitaxy. Parameters of investigated samples are given in Table I. The sample edges are oriented along the [1피 and [110] crystallographic axes. Eight pairs of contacts on each sample allow us to probe the photocurrent in different directions [see Fig. 4(b)].

To excite spin photocurrents we use the radiation of a high power pulsed terahertz molecular laser optically pumped by a TEA- $\mathrm{CO}_{2}$ laser. The linearly polarized radiation at a wavelength of $148 \mu \mathrm{m}$ with $10 \mathrm{~kW}$ power is modified to circular polarization by using a $\lambda / 4$ quartz plate. The terahertz radiation induces free carrier (Drude) absorption in the lowest conduction subband $e 1$. The photocurrent $j(\theta)$ is measured at room temperature in unbiased structures via the voltage drop across a $50 \Omega$ load resistor in a closed circuit configuration with a fast storage oscilloscope. ${ }^{5}$ The measured current pulses of $100 \mathrm{~ns}$ duration reflect the corresponding laser pulses.
In measurements of the spin-galvanic effect the samples are irradiated along the growth direction and an external magnetic field of $B=0.3 \mathrm{~T}$ is applied in the plane of the structure. In experiments on the circular photogalvanic effect we use oblique incidence and zero magnetic field. When investigating spin photocurrents one should take into account that optical excitation may generate other currents which are not the result of spin splitting and, simultaneously, are not sensitive to switching from right- to left-handed polarization. In the spin-galvanic effect setup magnetogyrotropic effects $^{13,32,33}$ may play an essential role and in the circular photogalvanic effect setup the linear photogalvanic effect and the photon drag may be of importance. These effects depend on the symmetry of the material in a different way than spin photocurrents. Therefore in most cases it is possible to choose a crystallographic orientation and an experimental geometry where only spin photocurrents occur. However, for the described experiments on Rashba/Dresselhaus spin-splitting measurements of the photocurrent in various crystallographic directions are needed. Therefore the total current results from the sum of different effects. In spite of this fact spin photocurrents can be recognized by their dependence on the helicity of the exciting radiation. Indeed, only spin photocurrents change their direction when the state of polarization of radiation is switched from right to left handed or vice versa. This allows one to extract the spin photocurrent contribution from the total photocurrent. Thus, we detect the photocurrent response for right $\left(\sigma_{+}\right)$and left $\left(\sigma_{-}\right)$handed circularly polarized radiation and extract spin photocurrents caused by spin splitting after eliminating current contributions which remain constant for $\sigma_{+}$and $\sigma_{-}$radiation. The helicity dependent photocurrent $j$ we discuss here is obtained as $j=\left(j_{\sigma_{+}}-j_{\sigma_{-}}\right) / 2$. We note that in most cases spin photocurrents are larger or of the same order of magnitude as the background current (see Refs. 5, 13, and 32) which makes this procedure possible.

\section{RESULTS AND DISCUSSION}

To obtain the RD ratio the spin photocurrents are measured for either a fixed orientation of the in-plane magnetic 

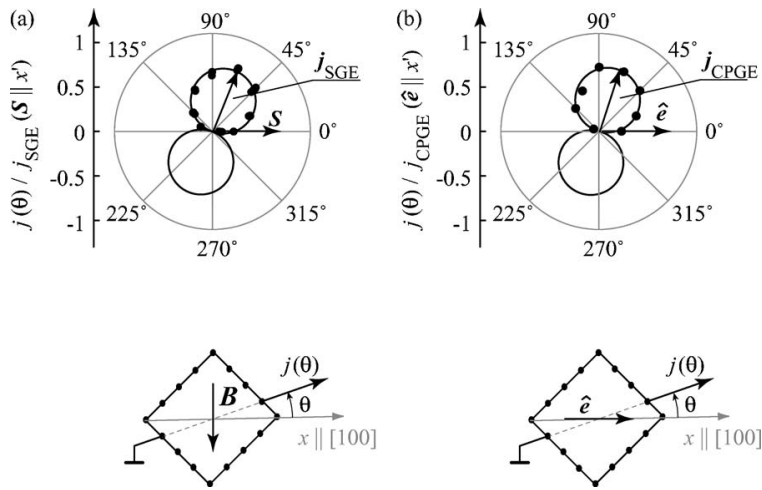

FIG. 5. Azimuthal dependences of spin-galvanic (a) and circular photogalvanic (b) photocurrents, $j(\theta)$. Data are obtained in an $n$-type InAs single QW (sample 1) applying SGE-I and CPGE-I geometries. The magnitude of the current measured at radiation power of $10 \mathrm{~kW}$ is normalized to the current maximum detected in the $y^{\prime}$ direction $\left[j_{\max }=j_{\mathrm{SGE}}\left(S \| x^{\prime}\right)\right]$ for SGE and $\left[j_{\max }\right.$ $\left.=j_{\text {CPGE }}\left(\hat{\boldsymbol{e}} \| x^{\prime}\right)\right]$, respectively. Bottom panels show geometries of corresponding experiments.

field in the case of the spin-galvanic effect or a fixed direction of light propagation in the case of the circular photogalvanic effect. Correspondingly $\boldsymbol{B}$ or $\hat{\boldsymbol{e}}$ are oriented along one of the three particular crystallographic directions, namely [100], [110], or [1 $\overline{10}$ ]. Then, the current is picked-up from the opposite contact pairs which yields a current distribution with respect to the crystallographic axes. The experimentally obtained currents measured in different directions, given by the angle $\theta$, are presented in polar coordinates.

\section{A. InAs low dimensional structures}

Figure 5(a) shows the result for a InAs QW structure obtained by the spin-galvanic effect for the magnetic field oriented along a cubic axis [010]. In the InAs QW structure investigated here, the Elliott-Yafet mechanism dominates spin relaxation ${ }^{34}$ which is isotropic. ${ }^{8}$ Thus the anisotropy of spin relaxation can be neglected and the in-plane average spin $\boldsymbol{S}$ of photoexcited carriers is perpendicular to $\boldsymbol{B}$. Therefore the ratio of Rashba and Dresselhaus currents can be directly read off from the left hand side of Fig. 5. Helicity dependent photocurrent flowing along the magnetic field, $j(\pi / 2)$, and therefore normal to the in-plane average spin is $j_{R}$ and the current measured in the direction perpendicular to the magnetic field $j(0)$ is $j_{D}$. The $\mathrm{RD}$ ratio $\alpha / \beta=j_{R} / j_{D}$ $=2.1 \pm 0.3$. Moreover, using this value for $\alpha / \beta$ all data on the Fig. 5(a) can be fitted by Eq. (12) by one fitting parameter corresponding to the strength of the current being independent of the angle $\theta$. From Fig. 5(a) it is seen that the current $j(\pi / 2)$ in the direction normal to the average spin is larger than $j(0)$. This unambiguously shows that the Rashba contribution dominates the spin splitting.

We also obtained the RD ratio applying the circular photogalvanic effect by oblique incidence of the radiation in a plane containing the [100] axis [Fig. 5(b)]. Figures 5(a) and 5(b) demonstrate the similarity of the results obtained by the different effects. The photogalvanic data can be fitted by Eq.

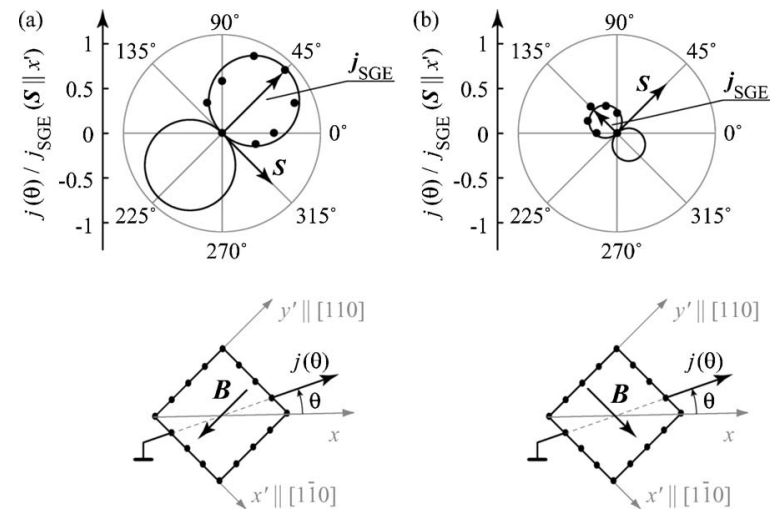

FIG. 6. Azimuthal dependences of spin-galvanic photocurrent measured in $n$-type InAs single QW (sample 1) applying SGE-II geometries. The magnitude of the current measured at radiation power of $10 \mathrm{~kW}$ is normalized to the current maximum $\left[j_{\max }\right.$ $\left.=j_{\mathrm{SGE}}\left(S \| x^{\prime}\right)=20 \mu \mathrm{A}\right]$ detected in the $y^{\prime}$ direction obtained in the geometry (a). Bottom panels show geometries of corresponding experiments.

(12), too, and yield almost the same ratio $j_{R} / j_{D}$ $=j(\pi / 2) / j(0)=\alpha^{\prime} / \beta^{\prime}=2.3$. The fact that the RD ratio obtained by CPGE is close to that extracted from the SGE may be attributed either to to always the dominating role of the first subband in the formation of the CPGE due to Drude absorption or to the overweighting contribution of the optically induced SGE. ${ }^{26}$ We applied this method to two other InAs structures. The growth condition of these samples were quite similar to the first one. Therefore, as expected, the Rashba to Dresselhaus spin-splitting ratios are obtained very close to each other (see Table I).

The geometry of the experiment shown so far is sufficient to extract the $\mathrm{RD}$ ratio and to conclude on the dominating mechanism of the spin splitting. However to demonstrate the self-consistency of the method we performed measurements in the geometry SGE-II as well. Figure 6 presents data obtained by the spin-galvanic effect. Applying the magnetic field along the $[110]$ or $[1 \overline{1} 0]$ directions we obtain current distributions plotted in Figs. 6(a) and 6(b). It is seen that, as expected from Eq. (4), for these geometries the current always reaches its maximum perpendicularly to the in-plane average spin. The magnitudes of the helicity dependent photocurrent in Figs. 6(a) and 6(b) are substantially different. Note that the data in Figs. 6(a) and 6(b) is normalized to $j_{\mathrm{SGE}}\left(\boldsymbol{S} \| x^{\prime}\right)=j(\pi / 4)$ measured in the geometry of Fig. 6(a). This is the maximum possible current because Rashba and Dresselhaus contributions point in the same direction in this geometry [Fig. 2(d)]. The ratio of Rashba and Dresselhaus currents can be evaluated from the currents $j(\pi / 4)$ in Fig. 6(a) $[j \propto(\alpha+\beta)]$ and $j(3 \pi / 4)$ in Fig. 6(b) $[j \propto(\alpha-\beta)]$. Knowing that the Rashba spin splitting is dominant, this procedure gives the same result as obtained by the first geometry: $j_{R} / j_{D}=2.1$ demonstrating the self-consistency of the method described. Investigation of the CPGE response in the similar geometry $(\hat{\boldsymbol{e}} \|\langle 110\rangle)$ gives the same result. As addressed above the dominance of the Rashba term cannot be deduced solely from these measurements.

The RD ratio of 1.6-2.3 agrees well with theoretical results ${ }^{35}$ which predict a dominating Rashba spin-orbit cou- 
pling for InAs QWs and is also consistent with experiments. $^{20,36}$ For InGaAs QWs, having similar sample parameters as the structures investigated here, $\alpha / \beta$ ratios were obtained from weak antilocalization experiments ${ }^{20}$ and $\boldsymbol{k} \cdot \boldsymbol{p}$ calculations. ${ }^{37}$ The corresponding values ranged between 1.5-1.7 and 1.85, respectively. These results are in good agreement with our findings. The RD ratio has previously been estimated from fits of magnetotransport and Hanle experiments. ${ }^{20,23,31}$ In contrast to these works our method allows to measure directly the relative strength of Rashba and Dresselhaus terms and does not require any additional theoretical estimations.

\section{B. GaAs low dimensional structures}

The RD ratio for GaAs quantum wells is obtained applying both SGE-I and CPGE-I methods. The results are presented in Table I. Measurements show that the Rashba constant $\alpha$ is larger than the Dresselhaus constant $\beta$ in all studied samples.

Let us first discuss the results for the single heterojunction. In CPGE measurements in geometry I one obtains $j(\pi / 2) / j(0)=7.6$ which yields consequently $\alpha / \beta=7.6$. The fact that in single heterojunctions the Rashba contribution is much larger is not surprising because, on the one hand, such structures, due to the triangular confinement potential, are strongly asymmetric and, on the other hand, the $\boldsymbol{k}$-linear Dresselhaus terms in wide structures such as those here are reduced. Measurements of the spin-galvanic effect in such structures give the ratio of the currents measured for a magnetic field along the $y$ axis: $j(\pi / 2) / j(0)=2.65$. It is well known that the dominant spin relaxation mechanism in GaAs is the D'yakonov-Perel' relaxation, therefore the RD ratio should be calculated applying the relevant Eqs. (16) and (17). We then obtain $\alpha / \beta=7.6$ which is in agreement with the value obtained from CPGE measurements and demonstrates applicability of the SGE method also for a dominating D'yakonov-Perel' mechanism (see Table I).

Now we compare spin splittings in the single heterojunction and the $8.2 \mathrm{~nm}$ wide QW. It is seen that the absolute value of the R/D-ratio is larger for the heterojunction than for the $\mathrm{QW}$, and the signs of the ratios are opposite. The change of the absolute value can be attributed to two facts. On the one hand, increase of the electron confinement increases the Dresselhaus constant $\beta$. On the other hand, SIA in the heterojunction is higher which means larger Rashba constant $\alpha$. Self-consistent solution of Schrödinger and Poisson equations for electron wave functions in our heterojunction and in the $8.2 \mathrm{~nm}$ wide QW shows that the wave function is indeed stronger localized in the QW than in the heterojunction. The relative sign of $\mathrm{RD}$ ratio is obtained from directions of the currents $\boldsymbol{j}_{R}$ and $\boldsymbol{j}_{D}$. Measurements show that the directions of the Dresselhaus current $\boldsymbol{j}_{D}$ in both samples remains the same, while the direction of the Rashba current $\boldsymbol{j}_{R}$ is reversed in the $8.2 \mathrm{~nm}$ wide QW. Conservation of the $\boldsymbol{j}_{D}$ sign is obvious because the sign of the Dresselhaus spin splitting is determined by the bulk properties of the material and, hence, is the same in both GaAs based systems. The reversing of $\boldsymbol{j}_{R}$, caused by SIA, follows from the fact that built-in electric fields, determining SIA, have opposite directions in these structures resulting in opposite signs of the Rashba constants $\alpha$.

In the 15 and $30 \mathrm{~nm}$ wide GaAs QWs we controllably varied the strength of SIA. This has been achieved by doping at different distances from the QW. For quantum wells of the same width, asymmetrical and symmetrical modulation doping yields larger and smaller strengths of SIA, respectively. At the same time for a given QW width, the strength of the Dresselhaus spin splitting is constant because it is independent of the doping position. Thus, variation of the doping distance should affect the RD ratio. Indeed, for more symmetric QW structures of both 15 and $30 \mathrm{~nm}$ width the ratio is smaller (see Table I). Opposite signs of the RD ratio for these samples as well as for previously discussed GaAs samples, are consistent with the direction of built-in electric fields obtained from solving Schrödinger and Poisson equations.

We emphasize that the observed sign reversal of the RD ratio upon changing of the $\delta$-doping plane in the heterostructure opens a way of QW growth with controllable spin splitting. Our results demonstrate that SIA can be tuned in a wide range with $\alpha$ both positive and negative. Measuring the Rashba photocurrents in the geometries SGE-I or CPGE-I as a function of doping position in structures of same width allows one to find the sample with zero Rashba photocurrent, corresponding to $\alpha=0$. Applying geometry SGE-II or CPGE-II for the sample one can find zero photocurrent which, according to Eqs. (6) and (10), means that the condition of equal Rashba and Dresselhaus constants ${ }^{10}(\alpha=\beta)$, important for spintronics applications, is realized.

\section{SUMMARY}

To summarize, we investigate Rashba and Dresselhaus spin-orbit splittings in various III-V material based 2D structures at room temperature applying spin photocurrents. We use the SGE and the CPGE and demonstrate self-consistency of the obtained results. In all investigated samples the Rashba contribution dominates over the Dresselhaus one. We emphasize that our measurements give the necessary feedback for structures with equal Rashba and Dresselhaus spin splittings or perfectly symmetric structures with zero Rashba constant.

\section{ACKNOWLEDGMENTS}

We thank E. L. Ivchenko and S. A. Tarasenko, as well as M. Koch and T. Kleine-Ostmann for helpful discussions. The high quality InAs quantum wells were kindly provided by $\mathrm{J}$. De Boeck and G. Borghs from IMEC Belgium. This work is supported by the Deutsche Forschungsgemeinschaft via Collaborative Research Center Grants Nos. SFB689 and GRK638, the Russian President grant for young scientists (RFBR), Russian Science Support Foundation (RAS), and Hans Böckler Stiftung. 
${ }^{1}$ I. Zutic, J. Fabian, and S. Das-Sarma, Rev. Mod. Phys. 76, 323 (2004).

${ }^{2}$ Y. A. Bychkov and E. I. Rashba, Pis'ma Zh. Eksp. Teor. Fiz. 39, 66 (1984) 39, 78 (1984)].

${ }^{3}$ M. I. D'yakonov and V. Yu. Kachorovskii, Fiz. Tekh. Poluprovodn. (S.-Peterburg) 20, 178 (1986) [Sov. Phys. Semicond. 20, 110 (1986)].

${ }^{4}$ G. Lommer, F. Malcher, and U. Rössler, Phys. Rev. B 32, 6965 (1985).

${ }^{5}$ S. D. Ganichev and W. Prettl, J. Phys.: Condens. Matter 15, R935 (2003), and references cited therein.

${ }^{6}$ F. G. Pikus and G. E. Pikus, Phys. Rev. B 51, 16928 (1995).

${ }^{7}$ N. S. Averkiev and L. E. Golub, Phys. Rev. B 60, 15582 (1999).

${ }^{8}$ N. S. Averkiev, L. E. Golub, and M. Willander, J. Phys.: Condens. Matter 14, R271 (2002).

${ }^{9}$ S. A. Tarasenko and N. S. Averkiev, Pis'ma Zh. Eksp. Teor. Fiz. 75, 669 (2002) [JETP Lett. 75, 552 (2002)].

${ }^{10}$ J. Schliemann, J. C. Egues, and D. Loss, Phys. Rev. Lett. 90, 146801 (2003).

${ }^{11}$ S. D. Ganichev, V. V. Bel'kov, L. E. Golub, E. L. Ivchenko, Petra Schneider, S. Giglberger, J. Eroms, J. De Boeck, G. Borghs, W. Wegscheider, D. Weiss, and W. Prettl, Phys. Rev. Lett. 92, 256601 (2004).

${ }^{12}$ S. D. Ganichev, E. L. Ivchenko, V. V. Bel'kov, S. A. Tarasenko, M. Sollinger, D. Weiss, W. Wegscheider, and W. Prettl, Nature (London) 417, 153 (2002).

${ }^{13}$ S. D. Ganichev and W. Prettl, Intense Terahertz Excitation of Semiconductors (Oxford University Press, Oxford, 2006).

${ }^{14}$ E. L. Ivchenko, Optical Spectroscopy of Semiconductor Nanostructures (Alpha Science, Harrow, 2005).

${ }^{15}$ S. D. Ganichev, E. L. Ivchenko, S. N. Danilov, J. Eroms, W. Wegscheider, D. Weiss, and W. Prettl, Phys. Rev. Lett. 86, 4358 (2001).

${ }^{16}$ M. Sasaki, Y. Ohno, and H. Ohno (unpublished).

${ }^{17}$ M. Bieler, N. Laman, H. M. van Driel, and A. L. Smirl, Appl. Phys. Lett. 86, 061102 (2005).

${ }^{18}$ C. L. Yang, H. T. He, Lu Ding, L. J. Cui, Y. P. Zeng, J. N. Wang, and W. K. Ge, Phys. Rev. Lett. 96, 186605 (2006).

${ }^{19}$ E. A. de Andrada e Silva, Phys. Rev. B 46, 1921 (1992).

${ }^{20}$ W. Knap, C. Skierbiszewski, A. Zduniak, E. Litwin-Staszewska, D. Bertho, F. Kobbi, J. L. Robert, G. E. Pikus, F. G. Pikus, S. V. Iordanskii, V. Mosser, K. Zekentes, and Yu. B. Lyanda-Geller, Phys. Rev. B 53, 3912 (1996).

${ }^{21}$ E. L. Ivchenko and G. E. Pikus, Superlattices and Other Heterostructures. Symmetry and Optical Phenomena (Springer, Berlin, 1997).

${ }^{22}$ B. Jusserand, D. Richards, G. Allan, C. Priester, and B. Etienne, Phys. Rev. B 51, 4707 (1995).
${ }^{23}$ J. B. Miller, D. M. Zumbühl, C. M. Marcus, Y. B. Lyanda-Geller, D. Goldhaber-Gordon, K. Campman, and A. C. Gossard, Phys. Rev. Lett. 90, 076807 (2003).

${ }^{24}$ A. Łusakowski, J. Wróbel, and T. Dietl, Phys. Rev. B 68, 081201 (2003).

${ }^{25}$ W. H. Lau and M. E. Flatté, Phys. Rev. B 72, 161311 (2005).

${ }^{26}$ We note that strictly speaking under oblique incidence the photocurrent is caused by a superposition of the CPGE and the SGE and the total current $\boldsymbol{j}=\boldsymbol{j}_{\mathrm{CPGE}}+\boldsymbol{j}_{\mathrm{SGE}}$ (Refs. 5,13 , and 15). The individual contributions can be separated in time-resolved experiments only. However, estimations show that for the photocurrent formation due to Drude absorption usually $j_{\mathrm{CPGE}}>j_{\mathrm{SGE}}$ which allows to neglect the SGE contribution (Refs. 13 and 27). Even if the SGE dominates the RD ratio can be determined from measurements under oblique incidence. In this case Eqs. (3)-(7) should be used taking into account that for optical spin orientation $\boldsymbol{S} \| \hat{\boldsymbol{e}}$.

${ }^{27}$ E. L. Ivchenko and S. A. Tarasenko (private communication).

${ }^{28}$ The measurements should be carried out at low magnetic field strengths so that $\omega_{L} \tau_{s}<1$ and, thus, the Hanle denominator in $S$ can be neglected. For higher magnetic fields the Hanle denominator must be taken into account.

${ }^{29}$ Here we neglect the anisotropy of the $g$ factor in the plane of the structure assuming it to be small, as it has been demonstrated in an asymmetric GaAs QWs of $10 \mathrm{~nm}$ width in Ref. 30 where the anisotropy was about ten percents.

${ }^{30}$ S. Hallstein, M. Oestreich, W. W. Rühle, and K. Köhler, in High Magnetic Fields in the Physics of Semiconductors II, edited by G. Landwehr and W. Ossau (World Scientific, Singapore, 1997), Vol. 2, p. 593.

${ }^{31}$ N. S. Averkiev, L. E. Golub, A. S. Gurevich, V. P. Evtikhiev, V. P. Kochereshko, A. V. Platonov, A. S. Shkolnik, and Yu. P. Efimov, Phys. Rev. B 74, 033305 (2006).

${ }^{32}$ V. V. Bel'kov, S. D. Ganichev, E. L. Ivchenko, S. A. Tarasenko, W. Weber, S. Giglberger, M. Olteanu, H.-P. Tranitz, S. N. Danilov, Petra Schneider, W. Wegscheider, D. Weiss, and W. Prettl, J. Phys.: Condens. Matter 17, 3405 (2005).

${ }^{33}$ S. D. Ganichev, V. V. Bel'kov, S. A. Tarasenko, S. N. Danilov, S. Giglberger, Ch. Hoffmann, E. L. Ivchenko, D. Weiss, W. Wegscheider, Ch. Gerl, D. Schuh, J. Stahl, J. De Boeck, G. Borghs, and W. Prettl, Nat. Phys. 2, 609 (2006).

${ }^{34}$ A. Takeuchi, T. Kuroda, S. Muto, and O. Wada, Physica B 272, 318 (1999)

${ }^{35}$ G. Lommer, F. Malcher, and U. Rössler, Phys. Rev. Lett. 60, 728 (1988).

${ }^{36}$ J. Nitta, T. Akazaki, H. Takayanagi, and T. Enoki, Phys. Rev. Lett. 78, 1335 (1997).

${ }^{37}$ P. Pfeffer and W. Zawadzki, Phys. Rev. B 59, R5312 (1999). 